\title{
VARIABILIDAD MORFOESTRUCTURAL DE OVINOS BLACKBELLY EN CAMPECHE, MÉXICO
}

\author{
MORPHOSTRUCTURAL VARIABILITY OF BLACKBELLY SHEEP IN CAMPECHE, MEXICO
}

\author{
Dzib, C.A. ${ }^{1}$, Ortiz de Montellano, A. ${ }^{1}$ y Torres-Hernández, G. ${ }^{2}$
}

${ }^{1}$ Instituto Tecnológico de Chiná. Campeche. México.

${ }^{2}$ Programa de Ganadería. Colegio de Postgraduados. Montecillo. Estado de México. México. glatohe@colpos.mx

\section{Palabras ClaVe adicionales}

Medidas corporales. Ovinos tropicales. Conservación. Caracterización racial.

\section{RESUMEN}

Con el propósito de estudiar la conformación corporal de ovinos Blackbelly, se tomó una muestra aleatoria de 220 hembras y 36 machos de los rebaños del municipio de Campeche, México. La zoometría fue la base del estudio y se evaluó con 14 medidas corporales y el peso vivo de ovinos de uno, dos y tres años de edad. Se efectuaron análisis de varianza y de correlación, así como un análisis de conglomerados en los machos y hembras de uno y tres años de edad. Las medidas corporales tuvieron cambios significativos $(p \leq 0,05)$ hasta los tres años edad. Las diferencias entre hembras y machos fueron más grandes después de un año de edad, sobre todo en el peso vivo. Todas las mediciones se correlacionaron significativamente $(p \leq 0,01)$, particularmente el peso vivo con el perímetro torácico, con la altura a la cruz, y con el perímetro testicular. Los animales de un año de edad mostraron una variabilidad corporal considerable; una parte de ellos tenía valores zoométricos muy cercanos a los límites máximos estimados en el grupo de tres años de edad. Por sus patrones corporales en la etapa adulta, los ovinos Blackbelly muestran una tendencia a ser animales productores de carne.

\section{SUMMARY}

In order to study the body conformation of Blackbelly sheep, a random sample of 220 females and 36 males was taken in flocks of the municipality of Campeche, Mexico. Zoometry was the base of the study and it was evaluated with 14 body

Recibido: 22-2-10. Aceptado: 8-6-11.

\section{AdDITIONAL KEYWORDS}

Body measurements. Tropical sheep. Conservation. Breed characterization.

measurements and live weight of one, two and three years old sheep. Analysis of variance and correlation were performed, as well as a clustering test in ewes and rams one and three years old. Body measurements had significant $(p \leq 0.05)$ changes throughout three years of age. Differences between males and females were higher after one year of age, especially live weight. All measurements were significantly correlated $(p \leq 0.01)$, particularly live weight with thoracic perimeter, height at withers, and testicular perimeter. Oneyear old animals had a considerable body variability; a portion of them had zoometric values very close to the maximum limits that were estimated in the three-year-old group. Based on their body patterns as adults, Blackbelly sheep show a tendency to be meat producer animals.

\section{INTRODUCCIÓN}

En las razas nativas y criollas es importante, entre otras acciones, conocer el tamaño de sus poblaciones, así como determinar su caracterización genética y fenotípica para establecer estrategias apropiadas para su conservación y aprovechamiento (Lastra, 1998). Alderson (1999) indicó que las medidas corporales simples tienen un uso relevante para los productores dentro de sus granjas y rebaños. Riva et al. (2004) mencionaron que la información proveniente de los animales para selección y el resultado de 
programas de mejoramiento genético pueden ser evaluados sobre una base morfológica. Este tipo de medidas ha tenido mucho uso en la caracterización de razas (Peña Blanco et al., 1990; Álvarez et al., 2000a; Álvarez et al., 2000b; Nsoso et al., 2004; Janssens y Vandepitte, 2004; Mernies et al., 2007; Kunene et al., 2007; Traoré et al., 2008), para analizar cambios en el tamaño y forma a través del tiempo (Arthur y Ahunu, 1989; Fasae et al., 2005), para establecer relaciones entre diversas medidas corporales (Otoikhian et al., 2008; Gusmão Filho et al., 2009), así como para predecir el peso vivo en función de medidas corporales (Thys y Hardouin, 1991; Afolayan et al., 2006; Costa Júnior et al., 2006; Sowande y Sobola, 2008). En los ovinos de pelo, recursos genéticos cada vez más importantes en las regiones tropicales de México, se han estudiado medidas corporales de ovinos Pelibuey (Castillo et al., 1974; Martínez Ávalos et al., 1987; Vilaboa y Díaz Rivera, 2007), así como en Katahdin y Dorper (Vilaboa y Díaz Rivera, 2007), pero no existe esta información en ovinos Blackbelly, que junto con los Pelibuey fueron las primeras razas de pelo introducidas en México y a la vez son las predominantes en el sureste de México, criadas bajo las mismas condiciones de manejo. La raza Blackbelly es originaria de África (Mason, 1988), y se ha popularizado por su rusticidad y alta prolificidad en los ambientes húmedos y subhúmedos, por lo que se encuentra muy bien adaptada a las estructuras productivas del sureste de México (González et al., 2002). Por tanto, su mejoramiento y conservación genética deben ser la base de sistemas productivos realmente sostenibles. Más aún, cuando aumenta la amenaza de pérdida de nuestras razas nativas, por el indiscriminado cruzamiento con las denominadas razas mejoradas (FAO, 2000). Con base en lo anterior, el objetivo de este trabajo fue determinar la variabilidad morfoestructural de ovinos Blackbelly a través de medidas y pesos corporales.

\section{MATERIAL Y MÉTODOS}

El trabajo se llevó a cabo con 220 hembras y 36 machos Blackbelly provenientes de 66 rebaños comerciales del Municipio de Campeche, en el Estado del mismo nombre, México. En virtud a las diferencias existentes que se habían encontrado en cuanto a los niveles de tecnificación (bajo, medio y alto) en esos rebaños (Dzib-Can et al., 2006), el muestreo de animales fue aleatorio. Esta muestra representó el $24 \%$ de las unidades de producción que tienen ovinos en el territorio municipal (INEGI, 1998).

La base del trabajo fue la zoometría, midiendo 14 variables zoométricas, así como el peso vivo de ovinos de uno, dos y tres años de edad, identificados mediante la evolución dentaria, de acuerdo a lo indicado por Devendra y Mcleroy (1982). En los animales se midieron: peso vivo, altura a la cruz, altura a la grupa, ancho del hombro (entre las escápulas), ancho del esternón (entre las axilas), altura al pecho (del suelo al esternón), profundidad del pecho (por diferencia entre altura a la cruz y altura al pecho, como medida del diámetro del cuerpo), longitud cruz-grupa (la grupa al nivel de las caderas), largo del lomo (la base ósea son las vértebras lumbares), ancho del lomo (entre las primeras vértebras lumbares), largo de la pierna (del isquion a la mitad de la tibia), ancho de la pierna (del isquion a la babilla; la babilla es el pliegue entre la pierna y vientre), perímetro torácico, perímetro de la caña, y perímetro testicular.

Después de comprobar las hipótesis de normalidad de los datos mediante la prueba de Shapiro-Wilk (Daniel, 1990), de homocedasticidad (o igualdad de varianzas) con la prueba de Levene (Levene, 1960) y de independencia de las muestras utilizando la prueba de rachas (Álvarez Cáceres, 2007) se efectuó un análisis de varianza para cada medida corporal y el peso vivo en función de la edad de los animales, así como un análisis de correlación de Pearson entre todas las variables. La comparación de me- 


\section{MEDIDAS CORPORALES DE OVINOS BLACKBELLY EN MÉXICO}

dias se efectuó por medio de la prueba de Tukey, con un nivel de significancia de 0,05 . Adicionalmente se efectuó un análisis de conglomerados (Jonson, 2000) en los ovinos de un año de edad, con el propósito de ordenarlos en tres clases en función de sus medidas corporales, así como también en hembras y machos de uno y tres años de edad.

\section{RESULTADOSYDISCUSIÓN}

Excepto la altura a la grupa en machos (tabla I) y el ancho del esternón y perímetro de la caña en hembras (tabla II), las medidas zoométricas y el peso vivo de machos y hembras aumentaron con la edad $(\mathrm{p} \leq 0,05)$. Machos y hembras de un año de edad (tabla III) solamente difirieron en la longitud cruzgrupa, siendo más largos los machos. A los dos años de edad los machos superaron a las hembras $(\mathrm{p} \leq 0,05)$ en peso vivo, altura a la cruz, altura a la grupa, perímetro torácico, profundidad del pecho y longitud cruz-gru- pa. A los tres años de edad, además de las diferencias entre ambos sexos expresadas a los dos años de edad, se sumaron el ancho del lomo y de la pierna (tabla III). Valores más grandes en machos que en hembras a edad similar han sido reportados por Mehta et al. (1979) en ovinos de la India y Musa et al. (2005) en ovinos de África.

$\mathrm{Si}$ se considera un peso adulto de $52,0 \mathrm{~kg}$ a los tres años de edad en los machos (tabla I), los animales de un año de edad habían alcanzado el $60 \%$ de ese peso, además el $82 \%$ del perímetro testicular, el $90 \%$ de la altura a la cruz, el 85\% del perímetro torácico, el $95 \%$ de la longitud cruz-grupa y el $88 \%$ del perímetro de la caña. Searle et al. (1989) indicaron que las dimensiones esqueléticas de los ovinos se incrementan a mayor velocidad en el primer año de vida, especialmente la longitud corporal y altura a la cruz.

De acuerdo con el peso vivo máximo $(35,4 \mathrm{~kg})$ observado en las hembras de tres años de edad (tabla II), las ovejas de un año de edad habían logrado el $80 \%$ de ese peso

Tabla I. Media y desviación estándar de medidas corporales por edad en machos Blackbelly. (Mean and standard deviation of body measurements by age in Blackbelly rams).

\begin{tabular}{|c|c|c|c|}
\hline & $1(n=14)$ & $\begin{array}{c}\text { Edad, años } \\
2(n=10)\end{array}$ & $3(n=12)$ \\
\hline Peso vivo (kg) & $30,1 \pm 7,0^{a}$ & $42,0 \pm 6,3^{b}$ & $52,0 \pm 8,8^{c}$ \\
\hline Perímetro testicular (cm) & $24,7 \pm 4,0^{a}$ & $29,7 \pm 2,9^{b}$ & $30,1 \pm 1,9^{b}$ \\
\hline Altura a la cruz $(\mathrm{cm})$ & $64,2 \pm 5,2^{a}$ & $69,4 \pm 5,4^{b}$ & $71,5 \pm 5,3^{b}$ \\
\hline Altura a la grupa (cm) & $65,5 \pm 4,7$ & $69,4 \pm 4,7$ & $69,2 \pm 7,4$ \\
\hline Ancho del hombro (cm) & $15,0 \pm 2,7^{a}$ & $17,6 \pm 2,2^{a b}$ & $18,2 \pm 2,3^{b}$ \\
\hline Ancho del esternón (cm) & $8,6 \pm 1,2^{\mathrm{a}}$ & $9,2 \pm 1,4^{a}$ & $10,3 \pm 1,7^{\mathrm{ab}}$ \\
\hline Perímetro torácico (cm) & $73,3 \pm 7,9^{a}$ & $81,9 \pm 9,2^{b}$ & $86,5 \pm 6,5^{\mathrm{ab}}$ \\
\hline Altura al pecho $(\mathrm{cm})$ & $35,1 \pm 3,3^{a}$ & $37,5 \pm 3,9^{a b}$ & $38,1 \pm 1,8^{b}$ \\
\hline Profundidad del pecho (cm) & $28,8 \pm 4,4^{a}$ & $31,9 \pm 3,0^{a b}$ & $33,3 \pm 4,1^{b}$ \\
\hline Longitud cruz-grupa $(\mathrm{cm})$ & $39,8 \pm 4,1^{a}$ & $45,9 \pm 5,4^{b}$ & $42,0 \pm 6,0^{\mathrm{ab}}$ \\
\hline Largo del lomo (cm) & $16,1 \pm 1,2^{\mathrm{a}}$ & $17,7 \pm 1,3^{b}$ & $18,4 \pm 1,3^{b}$ \\
\hline Ancho del lomo (cm) & $12,6 \pm 1,6^{a}$ & $14,5 \pm 1,5^{a b}$ & $15,5 \pm 1,8^{b}$ \\
\hline Largo de la pierna $(\mathrm{cm})$ & $29,2 \pm 2,7^{a}$ & $31,8 \pm 3,2^{a b}$ & $33,2 \pm 2,4^{b}$ \\
\hline Ancho de la pierna (cm) & $21,3 \pm 2,2^{a}$ & $23,1 \pm 2,8^{a b}$ & $24,9 \pm 2,6^{b}$ \\
\hline Perímetro de la caña $(\mathrm{cm})$ & $7,8 \pm 0,8^{a}$ & $8,5 \pm 0,8^{a b}$ & $8,8 \pm 0,4^{b}$ \\
\hline$\%$ en la muestra & 38,8 & 27,7 & 33,3 \\
\hline
\end{tabular}

abc Literales distintas en hileras indican diferencias estadísticas $(p \leq 0,05)$. 


\section{DZIB, ORTIZ DE MONTELLANO Y TORRES-HERNÁNDEZ}

Tabla II. Media y desviación estándar de medidas corporales por edad en hembras Blackbelly. (Mean and standard deviation of body measurements by age in Blackbelly ewes).

\begin{tabular}{lccc}
\hline & & Edad, años \\
& $1(\mathrm{n}=44)$ & $2(\mathrm{n}=86)$ & $3(\mathrm{n}=90)$ \\
\hline Peso vivo $(\mathrm{kg})$ & $27,9 \pm 6,3^{\mathrm{a}}$ & $32,3 \pm 6,0^{\mathrm{b}}$ & $35,4 \pm 6,5^{\mathrm{c}}$ \\
Altura a la cruz $(\mathrm{cm})$ & $61,9 \pm 4,6^{\mathrm{a}}$ & $63,0 \pm 4,2^{\mathrm{a}}$ & $66,0 \pm 4,3^{\mathrm{b}}$ \\
Altura a la grupa $(\mathrm{cm})$ & $63,1 \pm 4,1^{\mathrm{a}}$ & $63,3 \pm 4,4^{\mathrm{a}}$ & $66,3 \pm 3,9^{\mathrm{b}}$ \\
Ancho del hombro $(\mathrm{cm})$ & $15,2 \pm 1,6^{\mathrm{a}}$ & $15,9 \pm 1,7^{\mathrm{ab}}$ & $16,5 \pm 2,2^{\mathrm{b}}$ \\
Ancho del esternón (cm) & $8,3 \pm 1,0$ & $8,7 \pm 1,0$ & $8,9 \pm 1,2$ \\
Perímetro torácico $(\mathrm{cm})$ & $71,1 \pm 6,4^{\mathrm{a}}$ & $73,1 \pm 6,3^{\mathrm{a}}$ & $77,7 \pm 6,1^{\mathrm{b}}$ \\
Altura al pecho $(\mathrm{cm})$ & $35,1 \pm 3,9^{\mathrm{a}}$ & $35,3 \pm 3,1^{\mathrm{a}}$ & $36,6 \pm 2,6^{\mathrm{b}}$ \\
Profundidad del pecho $(\mathrm{cm})$ & $26,8 \pm 2,5^{\mathrm{a}}$ & $27,7 \pm 3,8^{\mathrm{a}}$ & $29,4 \pm 3,5^{\mathrm{b}}$ \\
Longitud cruz-grupa $(\mathrm{cm})$ & $37,1 \pm 3,1^{\mathrm{a}}$ & $39,9 \pm 3,8^{\mathrm{b}}$ & $39,4 \pm 3,2^{\mathrm{b}}$ \\
Largo del lomo $(\mathrm{cm})$ & $16,8 \pm 1,6^{\mathrm{a}}$ & $17,4 \pm 1,4^{\mathrm{ab}}$ & $17,7 \pm 1,6^{\mathrm{b}}$ \\
Ancho del lomo $(\mathrm{cm})$ & $12,5 \pm 1,3^{\mathrm{a}}$ & $13,3 \pm 1,7^{\mathrm{b}}$ & $12,8 \pm 1,3^{\mathrm{a}}$ \\
Largo de la pierna $(\mathrm{cm})$ & $29,6 \pm 3,1^{\mathrm{a}}$ & $29,7 \pm 3,1^{\mathrm{a}}$ & $30,9 \pm 2,5^{\mathrm{b}}$ \\
Ancho de la pierna $(\mathrm{cm})$ & $21,4 \pm 2,3^{\mathrm{a}}$ & $23,2 \pm 3,2^{\mathrm{b}}$ & $22,1 \pm 2,2^{\mathrm{a}}$ \\
Perímetro de la caña $(\mathrm{cm})$ & $7,2 \pm 0,5$ & $7,4 \pm 0,6$ & $7,4 \pm 0,6$ \\
\% en la muestra & 20,0 & 39,1 & 40,9 \\
\hline
\end{tabular}

abciterales distintas en hileras indican diferencias estadísticas $(p<0,05)$.

Tabla III. Media y desviación estándar de medidas corporales por edad entre machos y hembras Blackbelly. (Mean and standard deviation of body measurements by age between Blackbelly rams and ewes).

\begin{tabular}{|c|c|c|c|c|c|c|}
\hline & \multicolumn{6}{|c|}{ Edad, años } \\
\hline & $\begin{array}{l}1(n=14) \\
\text { machos }\end{array}$ & $\begin{array}{l}1(n=44) \\
\text { hembras }\end{array}$ & $\begin{array}{c}2(n=10) \\
\text { machos }\end{array}$ & $\begin{array}{l}2(n=86) \\
\text { hembras }\end{array}$ & $\begin{array}{l}3(n=12) \\
\text { machos }\end{array}$ & $\begin{array}{l}3(n=90) \\
\text { hembras }\end{array}$ \\
\hline Peso vivo (kg) & $30,1 \pm 7,0$ & $27,9 \pm 6,3$ & $42,0 \pm 6,3^{\mathrm{a}}$ & $32,3 \pm 6,0^{b}$ & $52,0 \pm 8,8^{a}$ & $35,4 \pm 6,5^{b}$ \\
\hline Perímetro testicular $(\mathrm{cm})$ & $24,7 \pm 4,0$ & - & $29,7 \pm 2,9$ & - & $30,1 \pm 1,9$ & - \\
\hline Altura a la cruz $(\mathrm{cm})$ & $64,2 \pm 5,2$ & $61,9 \pm 4,6$ & $69,4 \pm 5,4^{\mathrm{a}}$ & $63,0 \pm 4,2^{b}$ & $71,5 \pm 5,3^{\mathrm{a}}$ & $66,0 \pm 4,3^{b}$ \\
\hline Altura a la grupa $(\mathrm{cm})$ & $65,5 \pm 4,7$ & $63,1 \pm 4,1$ & $69,4 \pm 4,7^{a}$ & $63,3 \pm 4,4^{b}$ & $69,2 \pm 7,4^{a}$ & $66,3 \pm 3,9^{b}$ \\
\hline Ancho del hombro (cm) & $15,0 \pm 2,7$ & $15,2 \pm 1,6$ & $17,6 \pm 2,2$ & $15,9 \pm 1,7$ & $18,2 \pm 2,3$ & $16,5 \pm 2,2$ \\
\hline Ancho del esternón (cm) & $8,6 \pm 1,2$ & $8,3 \pm 1,0$ & $9,2 \pm 1,4$ & $8,7 \pm 1,0$ & $10,3 \pm 1,7$ & $8,9 \pm 1,2$ \\
\hline Perímetro torácico $(\mathrm{cm})$ & $73,3 \pm 7,9$ & $71,1 \pm 6,4$ & $81,9 \pm 9,2^{\mathrm{a}}$ & $73,1 \pm 6,3^{b}$ & $86,5 \pm 6,5^{a}$ & $77,7 \pm 6,1^{\mathrm{b}}$ \\
\hline Altura al pecho $(\mathrm{cm})$ & $35,1 \pm 3,3$ & $35,1 \pm 3,9$ & $37,5 \pm 3,9$ & $35,3 \pm 3,1$ & $38,1 \pm 1,8$ & $36,6 \pm 2,6$ \\
\hline Profundidad del pecho $(\mathrm{cm})$ & $28,8 \pm 4,4$ & $26,8 \pm 2,5$ & $31,9 \pm 3,0^{a}$ & $27,7 \pm 3,8^{b}$ & $33,3 \pm 4,1^{a}$ & $29,4 \pm 3,5^{b}$ \\
\hline Longitud cruz-grupa (cm) & $39,8 \pm 4,1^{a}$ & $37,1 \pm 3,1^{b}$ & $45,9 \pm 5,4^{a}$ & $39,9 \pm 3,8^{b}$ & $42,0 \pm 6,0^{a}$ & $39,4 \pm 3,2^{b}$ \\
\hline Largo del lomo $(\mathrm{cm})$ & $16,1 \pm 1,2$ & $16,8 \pm 1,6$ & $17,7 \pm 1,3$ & $17,4 \pm 1,4$ & $18,4 \pm 1,3$ & $17,7 \pm 1,6$ \\
\hline Ancho del lomo $(\mathrm{cm})$ & $12,6 \pm 1,6$ & $12,5 \pm 1,3$ & $14,5 \pm 1,5$ & $13,3 \pm 1,7$ & $15,5 \pm 1,8^{a}$ & $12,8 \pm 1,3^{b}$ \\
\hline Largo de la pierna $(\mathrm{cm})$ & $29,2 \pm 2,7$ & $29,6 \pm 3,1$ & $31,8 \pm 3,2$ & $29,7 \pm 3,1$ & $33,2 \pm 2,4$ & $30,9 \pm 2,5$ \\
\hline Ancho de la pierna $(\mathrm{cm})$ & $21,3 \pm 2,2$ & $21,4 \pm 2,3$ & $23,1 \pm 2,8$ & $23,2 \pm 3,2$ & $24,9 \pm 2,6^{a}$ & $22,1 \pm 2,2^{b}$ \\
\hline Perímetro de la caña $(\mathrm{cm})$ & $7,8 \pm 0,8$ & $7,2 \pm 0,5$ & $8,5 \pm 0,8$ & $7,4 \pm 0,6$ & $8,8 \pm 0,4$ & $7,4 \pm 0,6$ \\
\hline
\end{tabular}

Archivos de zootecnia vol. 60, núm. 232, p. 1294. 
MEDIDAS CORPORALES DE OVINOS BLACKBELLY EN MÉXICO

Tabla IV. Correlaciones fenotípicas entre medidas corporales de machos Blackbelly. (Phenotypic correlations between body measurements of Blackbelly rams).

\begin{tabular}{|c|c|c|c|c|c|c|c|c|c|c|c|c|c|c|}
\hline & $A C$ & $A G$ & $\mathrm{AH}$ & $A E$ & ALP & PP & LCG & LL & $A L$ & LP & AP & PTO & PC & PTE \\
\hline PV & $\underset{* \star}{0,70}$ & $\underset{* *}{0,47}$ & $\underset{* *}{0,68}$ & $\underset{\star \star *}{0,42}$ & $\underset{* \star}{0,49}$ & $\begin{array}{c}0,60 \\
* *\end{array}$ & $\begin{array}{c}0,15 \\
\text { NS }\end{array}$ & $\begin{array}{c}0,61 \\
* *\end{array}$ & $\underset{* *}{0,55}$ & $\underset{* *}{0,70}$ & $\begin{array}{c}0,67 \\
* *\end{array}$ & $\underset{* *}{0,77}$ & $\underset{* *}{0,65}$ & $\underset{* \star}{0,64}$ \\
\hline$A C$ & - & $\underset{* *}{0,71}$ & $\underset{* *}{0,73}$ & $\begin{array}{c}0,41 \\
*\end{array}$ & $\begin{array}{c}0,73 \\
* *\end{array}$ & $\underset{* *}{0,85}$ & $\begin{array}{c}0,01 \\
\text { NS }\end{array}$ & $\underset{* *}{0,43}$ & $\begin{array}{c}0,47 \\
\text { NS }\end{array}$ & $\begin{array}{c}0,69 \\
* *\end{array}$ & $\begin{array}{c}0,40 \\
*\end{array}$ & $\begin{array}{c}0,84 \\
\star \star\end{array}$ & $\begin{array}{c}0,60 \\
* \star\end{array}$ & $\underset{* \star}{0,62}$ \\
\hline AG & $\begin{array}{l}- \\
-\end{array}$ & $\begin{array}{l}- \\
-\end{array}$ & $\begin{array}{l}0,41 \\
\text { * }\end{array}$ & $\begin{array}{c}0,07 \\
\text { NS }\end{array}$ & $\begin{array}{c}0,55 \\
\star *\end{array}$ & $\underset{* *}{0,57}$ & $\begin{array}{c}0,25 \\
\text { NS }\end{array}$ & $\begin{array}{c}0,09 \\
\text { NS }\end{array}$ & $\begin{array}{c}0,09 \\
\text { NS }\end{array}$ & $\underset{* \star}{0,51}$ & $\begin{array}{l}0,10 \\
\text { NS }\end{array}$ & $\underset{* \star}{0,54}$ & $\begin{array}{c}0,47 \\
\star *\end{array}$ & $\begin{array}{c}0,38 \\
*\end{array}$ \\
\hline $\mathrm{AH}$ & $\begin{array}{l}- \\
-\end{array}$ & $\begin{array}{l}- \\
-\end{array}$ & - & $\begin{array}{c}0,38 \\
*\end{array}$ & $\begin{array}{c}0,39 \\
*\end{array}$ & $\underset{* *}{0,73}$ & $\begin{array}{c}-0,10 \\
\text { NS }\end{array}$ & $\underset{* *}{0,47}$ & $\begin{array}{c}0,47 \\
* *\end{array}$ & $\underset{* *}{0,60}$ & $\begin{array}{c}0,34 \\
*\end{array}$ & $\underset{* *}{0,73}$ & $\begin{array}{c}0,47 \\
* *\end{array}$ & $\underset{* *}{0,65}$ \\
\hline$A E$ & - & $\begin{array}{l}- \\
-\end{array}$ & - & $\begin{array}{l}- \\
-\end{array}$ & $\underset{* *}{0,43}$ & $\begin{array}{c}0,25 \\
\text { NS }\end{array}$ & $\begin{array}{c}-0,10 \\
\text { NS }\end{array}$ & $\underset{* *}{0,59}$ & $\underset{* *}{0,40}$ & $\underset{* *}{0,57}$ & $\begin{array}{c}0,21 \\
\text { NS }\end{array}$ & $\begin{array}{c}0,44 \\
* *\end{array}$ & $\begin{array}{c}0,18 \\
\text { NS }\end{array}$ & $\begin{array}{c}0,41 \\
*\end{array}$ \\
\hline ALP & $\begin{array}{l}- \\
-\end{array}$ & $\begin{array}{l}- \\
-\end{array}$ & - & $\begin{array}{l}- \\
-\end{array}$ & $\begin{array}{l}- \\
-\end{array}$ & $\begin{array}{c}0,26 \\
\text { NS }\end{array}$ & $\begin{array}{c}-0,11 \\
\text { NS }\end{array}$ & $\begin{array}{c}0,24 \\
\text { NS }\end{array}$ & $\begin{array}{c}0,28 \\
N S\end{array}$ & $\underset{* *}{0,61}$ & $\begin{array}{c}0,19 \\
\text { NS }\end{array}$ & $\underset{* *}{0,49}$ & $\underset{\star * *}{0,27}$ & $\underset{* \star}{0,46}$ \\
\hline PP & - & - & - & $\begin{array}{l}- \\
-\end{array}$ & - & - & $\begin{array}{c}0,10 \\
\text { NS }\end{array}$ & $\underset{* *}{0,42}$ & $\underset{* *}{0,45}$ & $\underset{* *}{0,51}$ & $\underset{* *}{0,42}$ & $\underset{* *}{0,81}$ & $\underset{* *}{0,62}$ & $\underset{* *}{0,53}$ \\
\hline LCG & $\begin{array}{l}- \\
-\end{array}$ & $\begin{array}{l}- \\
-\end{array}$ & - & $\begin{array}{l}- \\
-\end{array}$ & - & - & $\begin{array}{l}- \\
-\end{array}$ & $\begin{array}{c}0,23 \\
\text { NS }\end{array}$ & $\begin{array}{l}0,19 \\
\text { NS }\end{array}$ & $\begin{array}{c}0,01 \\
\text { NS }\end{array}$ & $\underset{\star \star *}{0,42}$ & $\begin{array}{c}0,11 \\
\text { NS }\end{array}$ & $\begin{array}{c}0,44 \\
* *\end{array}$ & $\begin{array}{c}0,10 \\
\text { NS }\end{array}$ \\
\hline LL & - & - & - & - & - & - & - & - & $\underset{* *}{0,51}$ & $\underset{* *}{0,58}$ & $\underset{* \star}{0,47}$ & $\underset{* *}{0,59}$ & $\begin{array}{c}0,37 \\
*\end{array}$ & $\underset{* \star}{0,55}$ \\
\hline$A L$ & - & - & - & $\begin{array}{l}- \\
-\end{array}$ & - & - & - & - & - & $\begin{array}{c}0,40 \\
*\end{array}$ & $\underset{\star \star *}{0,63}$ & $\begin{array}{c}0,43 \\
\star *\end{array}$ & $\begin{array}{c}0,61 \\
\star \star\end{array}$ & $\begin{array}{c}0,37 \\
*\end{array}$ \\
\hline LP & - & - & - & - & - & - & - & - & $\begin{array}{l}- \\
-\end{array}$ & $\begin{array}{l}- \\
-\end{array}$ & $\begin{array}{c}0,37 \\
*\end{array}$ & $\begin{array}{c}0,70 \\
* *\end{array}$ & $\underset{* *}{0,45}$ & $\underset{* *}{0,76}$ \\
\hline AP & - & - & - & $\begin{array}{l}- \\
-\end{array}$ & - & - & - & - & - & - & - & $\begin{array}{c}0,48 \\
* *\end{array}$ & $\underset{* \star}{0,65}$ & $\begin{array}{c}0,28 \\
\text { NS }\end{array}$ \\
\hline PTO & - & - & - & $\begin{array}{l}- \\
-\end{array}$ & - & - & - & - & - & - & - & - & $\underset{\star \star *}{0,60}$ & $\underset{* *}{0,62}$ \\
\hline PC & - & - & - & - & - & - & - & - & - & - & - & - & - & $\underset{* *}{0,43}$ \\
\hline
\end{tabular}

PV: peso vivo; AC: altura a la cruz; AG: altura a la grupa; AH: ancho del hombro; AE: ancho del esternón; ALP: altura al pecho; PP: profundidad del pecho; LCG: longitud cruz-grupa; LL: largo del lomo; AL: ancho del lomo; LP: largo de la pierna; AP: ancho de la pierna; PTO: perímetro torácico; PC: perímetro de la caña; PTE: perímetro testicular; ${ }^{*} \mathrm{p} \leq 0,05 ;{ }^{*} \mathrm{p} \leq 0,01$; NS: no significativo.

maduro, además, el 94\% de la altura a la cruz, el 91\% del perímetro torácico, el 94\% de la longitud cruz-grupa y el $97 \%$ del perímetro de la caña, observación también de acuerdo con Searle et al. (1989).

Las diferencias entre machos y hembras fueron más grandes a partir de dos años de edad (tabla III). En referencia al peso vivo, los machos de uno, dos y tres años fueron 7,$3 ; 23$ y $31 \%$ más pesados que las hembras a las mismas edades. En la altura a la cruz, las diferencias a favor de los machos fueron de 3,$2 ; 9,2$ y $12,1 \%$, y en la longitud cruz-grupa los machos midieron 6,$8 ; 13,0$ y $6,1 \%$ más que las hembras. Estos resultados muestran que la dinámica del crecimiento es similar entre hembras y machos, aunque las hembras alcanzan el peso maduro más temprano, a esto se atribuye que las diferencias entre ambos sexos son más notorias después del año de edad. Otros estudios (Meraz et al., 1997; González et al., 2002) señalan 


\section{DZIB, ORTIZ DE MONTELLANO Y TORRES-HERNÁNDEZ}

que las diferencias en la curva de crecimiento entre hembras y machos se inician a los 100 días de edad, y son más notables a partir de los 180 días de edad, debido principalmente a efectos hormonales de la pubertad.

Con relación a la zoometría, los ovinos Blackbelly no mostraron diferencias notables con la zoometría del ovino Pelibuey de otras regiones de México, según lo informaron Ruz (1966) en Tabasco, Martínez Ávalos et al. (1987) y Ortiz et al. (2000) en Yucatán, Castillo et al. (1974) y Vilaboa y Díaz Rivera
(2007) en Veracruz. Las hembras Blackbelly de 3 años de edad superan ligeramente a las Pelibuey de Cuba de la misma edad en peso vivo, perímetro torácico y altura a la cruz (Pavon et al., 1986). Sin embargo, las hembras Pelibuey de Veracruz, México (Vilaboa y Diaz Rivera, 2007) superan a las Blackbelly de este estudio en peso corporal, perímetro torácico, y perímetro de la caña, aunque dichas medidas en las Pelibuey fueron tomadas a los 4 años 7 meses, mientras que en las Blackbelly a los 3 años. Asimismo, las

Tabla $\boldsymbol{V}$. Correlaciones fenotipicas entre medidas corporales de hembras Blackbelly. (Phenotypic correlations between body measurements of Blackbelly ewes).

\begin{tabular}{|c|c|c|c|c|c|c|c|c|c|c|c|c|c|}
\hline & $A C$ & $A G$ & $\mathrm{AH}$ & $A E$ & ALP & PP & LCG & LL & $A L$ & LP & AP & PTO & PC \\
\hline PV & $\underset{* \star}{0,60}$ & $\underset{* \star}{0,64}$ & $\underset{* *}{0,52}$ & $\underset{* *}{0,36}$ & $\underset{* *}{0,29}$ & $\underset{* *}{0,47}$ & $\underset{* *}{0,29}$ & $\underset{* *}{0,42}$ & $\begin{array}{c}0,11 \\
\text { NS }\end{array}$ & $\underset{* \star}{0,43}$ & $\underset{* \star}{0,24}$ & $\underset{* *}{0,82}$ & $\underset{* *}{0,41}$ \\
\hline$A C$ & - & $\underset{* *}{0,83}$ & $\begin{array}{c}0,31 \\
* *\end{array}$ & $\begin{array}{c}0,17 \\
*\end{array}$ & $\underset{* *}{0,62}$ & $\underset{* *}{0,72}$ & $\underset{\star *}{0,25}$ & $\underset{* \star}{0,31}$ & $\begin{array}{l}0,10 \\
\text { NS }\end{array}$ & $\underset{* \star}{0,41}$ & $\begin{array}{c}-0,02 \\
\text { NS }\end{array}$ & $\underset{* *}{0,59}$ & $\begin{array}{c}0,19 \\
* *\end{array}$ \\
\hline AG & $\begin{array}{l}- \\
-\end{array}$ & $\begin{array}{l}- \\
-\end{array}$ & $\begin{array}{c}0,40 \\
* *\end{array}$ & $\underset{* \star}{0,26}$ & $\underset{* *}{0,49}$ & $\underset{* *}{0,62}$ & $\begin{array}{c}0,11 \\
\text { NS }\end{array}$ & $\underset{* *}{0,33}$ & $\begin{array}{c}-0,01 \\
\text { NS }\end{array}$ & $\begin{array}{c}0,50 \\
* *\end{array}$ & $\begin{array}{c}-0,05 \\
\text { NS }\end{array}$ & $\underset{* \star}{0,66}$ & $\underset{* *}{0,23}$ \\
\hline $\mathrm{AH}$ & - & $\begin{array}{l}- \\
-\end{array}$ & - & $\underset{* *}{0,34}$ & $\begin{array}{c}0,15 \\
*\end{array}$ & $\underset{* *}{0,25}$ & $\begin{array}{c}0,21 \\
* *\end{array}$ & $\begin{array}{c}0,30 \\
* *\end{array}$ & $\begin{array}{c}0,17 \\
\star \star\end{array}$ & $\begin{array}{c}0,21 \\
\star \star\end{array}$ & $\begin{array}{c}0,15 \\
*\end{array}$ & $\begin{array}{c}0,45 \\
* *\end{array}$ & $\begin{array}{c}0,21 \\
* *\end{array}$ \\
\hline $\mathrm{AE}$ & $\begin{array}{l}- \\
-\end{array}$ & $\begin{array}{l}- \\
-\end{array}$ & $\begin{array}{l}- \\
-\end{array}$ & - & $\begin{array}{c}-0,06 \\
\text { NS }\end{array}$ & $\underset{* *}{0,26}$ & $\begin{array}{c}0,25 \\
\star \star\end{array}$ & $\begin{array}{c}0,29 \\
* *\end{array}$ & $\begin{array}{c}-0,03 \\
\text { NS }\end{array}$ & $\begin{array}{c}0,12 \\
\text { NS }\end{array}$ & $\begin{array}{c}0,07 \\
\text { NS }\end{array}$ & $\begin{array}{c}0,40 \\
\star *\end{array}$ & $\begin{array}{c}0,14 \\
*\end{array}$ \\
\hline ALP & - & $\begin{array}{l}- \\
-\end{array}$ & - & - & $\begin{array}{l}- \\
-\end{array}$ & $\begin{array}{c}-0,10 \\
\text { NS }\end{array}$ & $\begin{array}{c}0,16 \\
*\end{array}$ & $\begin{array}{c}0,17 \\
*\end{array}$ & $\underset{* *}{0,25}$ & $\underset{\star * *}{0,34}$ & $\begin{array}{c}-0,01 \\
\text { NS }\end{array}$ & $\begin{array}{c}0,27 \\
* *\end{array}$ & $\begin{array}{c}0,05 \\
\text { NS }\end{array}$ \\
\hline$P P$ & - & $\begin{array}{l}- \\
-\end{array}$ & - & $\begin{array}{l}- \\
-\end{array}$ & - & $\begin{array}{l}- \\
-\end{array}$ & $\begin{array}{c}0,18 \\
\star \star\end{array}$ & $\begin{array}{c}0,25 \\
* *\end{array}$ & $\begin{array}{c}-0,10 \\
\text { NS }\end{array}$ & $\underset{* *}{0,21}$ & $\begin{array}{c}-0,01 \\
\text { NS }\end{array}$ & $\begin{array}{c}0,51 \\
* \star\end{array}$ & $\begin{array}{c}0,20 \\
* *\end{array}$ \\
\hline LCG & $\begin{array}{l}- \\
-\end{array}$ & $\begin{array}{l}- \\
-\end{array}$ & - & $\begin{array}{l}- \\
-\end{array}$ & - & - & - & $\underset{* *}{0,32}$ & $\begin{array}{c}0,19 \\
* *\end{array}$ & $\begin{array}{c}-0,09 \\
\text { NS }\end{array}$ & $\underset{* *}{0,26}$ & $\begin{array}{c}0,13 \\
*\end{array}$ & $\begin{array}{c}0,14 \\
*\end{array}$ \\
\hline LL & - & $\begin{array}{l}- \\
-\end{array}$ & - & - & - & - & - & $\begin{array}{l}- \\
-\end{array}$ & $\begin{array}{c}0,07 \\
\text { NS }\end{array}$ & $\begin{array}{c}0,28 \\
\star *\end{array}$ & $\begin{array}{c}0,11 \\
\text { NS }\end{array}$ & $\underset{* \star}{0,38}$ & $\begin{array}{c}0,10 \\
\text { NS }\end{array}$ \\
\hline$A L$ & - & $\begin{array}{l}- \\
-\end{array}$ & - & $\begin{array}{l}- \\
-\end{array}$ & - & - & $\begin{array}{l}- \\
-\end{array}$ & $\begin{array}{l}- \\
-\end{array}$ & - & $\begin{array}{c}0,12 \\
\text { NS }\end{array}$ & $\underset{* *}{0,32}$ & $\begin{array}{l}0,09 \\
\text { NS }\end{array}$ & $\underset{* *}{0,18}$ \\
\hline$\sqcup P$ & - & $\begin{array}{l}- \\
-\end{array}$ & - & - & - & - & - & - & - & $\begin{array}{l}- \\
-\end{array}$ & $\underset{* *}{0,27}$ & $\underset{* *}{0,39}$ & $\underset{* \star}{0,24}$ \\
\hline AP & $\begin{array}{l}- \\
-\end{array}$ & - & - & - & - & $\begin{array}{l}- \\
-\end{array}$ & - & - & $\begin{array}{l}- \\
-\end{array}$ & - & - & $\begin{array}{c}-0,01 \\
\text { NS }\end{array}$ & $\underset{* *}{0,32}$ \\
\hline PTO & - & - & - & - & - & - & - & - & - & - & - & - & $\underset{* *}{0,29}$ \\
\hline
\end{tabular}

PV: peso vivo; AC: altura a la cruz; AG: altura a la grupa; $A H$ : ancho del hombro; AE: ancho del esternón; ALP: altura al pecho; PP: profundidad del pecho; LCG: longitud cruz-grupa; LL: largo del lomo; AL: ancho del lomo; LP: largo de la pierna; AP: ancho de la pierna; PTO: perímetro torácico; PC: perímetro de la caña; ${ }^{*} \mathrm{p} \leq 0,05 ;{ }^{* *} \mathrm{p} \leq 0,01$; NS: no significativo.

Archivos de zootecnia vol. 60, núm. 232, p. 1296. 


\section{MEDIDAS CORPORALES DE OVINOS BLACKBELLY EN MÉXICO}

hembras Blackbelly de 3 años de edad superan a las hembras Pelibuey de Yucatán de 6 años de edad en peso corporal, altura a la cruz, perímetro torácico, y perímetro de la caña (Martínez Ávalos et al., 1987).

Las mediciones corporales se correlacionaron entre sí $(\mathrm{p} \leq 0,01)$ en machos (tabla IV) y en hembras (tabla V), lo que sugiere un crecimiento armónico y de dependencia entre estas medidas, lo que en conjunto puede medirse como el peso vivo. Las correlaciones más grandes $(\mathrm{p} \leq 0,01)$ se obtuvieron entre el peso vivo y el perímetro torácico (machos $\mathrm{r}=0,77$, hembras $\mathrm{r}=0,82$ ), entre el peso y la altura a la cruz (machos $\mathrm{r}=0,70$, hembras $r=0,60)$ y entre el peso y el perímetro testicular $(\mathrm{r}=0,64)$, lo que indica que al aumentar de peso, tanto machos como hembras son más altos y anchos, lo que podría indicar que su tendencia como animales adultos es hacia un animal productor de carne. La longitud cruz-grupa no estuvo correlacionada $(\mathrm{p} \geq 0,05)$ con el peso vivo $(\mathrm{r}=$ $0,15)$, mientras que en las hembras esa correlación $(\mathrm{r}=0,29)$ si fue significativa $(p \leq 0,01)$; esto nos dice que los machos a la vez que aumentan de peso también son más altos y anchos, pero se mantienen cortos, a diferencia de las hembras, que al aumentar de peso son más altas, anchas y largas. En ovinos Pelibuey (Castillo et al., 1974; Pavon et al., 1986; Vilaboa y Díaz Rivera, 2007), Santa Inês de Brasil (Costa Júnior et al., 2006), y Yankasa de África (Fasae et al., 2005; Afolayan et al., 2006)) se han obtenido correlaciones entre 0,60 y 0,98 para los mismos grupos de variables que en el presente estudio. Lo anterior sugiere la posibilidad de efectuar selección en algunas variables por medio de respuestas correlacionadas. Con base en este criterio, complementado con análisis de regresión, algunos

Tabla VI. Medias de medidas zoométricas de hembras y machos Blackbelly de un año de edad. (Means of zoometric measures of Blackbelly ewes and rams one year old).

\begin{tabular}{|c|c|c|c|c|c|c|}
\hline & & Hembras & & & Machos & \\
\hline & $1(n=11)$ & $2(n=19)$ & $3(n=14)$ & $1(n=1)$ & $2(n=7)$ & $3(n=6)$ \\
\hline Peso vivo (kg) & $35,1^{a}$ & $32,2^{b}$ & $23,6^{c}$ & $44,0^{a}$ & $33,1^{b}$ & $24,3^{c}$ \\
\hline Perímetro testicular (cm) & - & - & - & $28,0^{a}$ & $27,5^{a}$ & $20,7^{b}$ \\
\hline Altura a la cruz (cm) & $62,0^{\mathrm{b}}$ & $68,0^{\mathrm{a}}$ & $59,5^{b}$ & $66,0^{a}$ & $67,8^{a}$ & $59,1^{\mathrm{b}}$ \\
\hline Altura a la grupa (cm) & $63,2^{b}$ & $68,3^{a}$ & $61,0^{b}$ & $70,0^{a}$ & $68,8^{a}$ & $61,0^{b}$ \\
\hline Ancho del hombro (cm) & 16,1 & 15,6 & 14,8 & $23,0^{a}$ & $15,2^{b}$ & $13,3^{b}$ \\
\hline Ancho del esternón (cm) & 9,1 & 8,3 & 8,1 & 9,0 & 9,0 & 8,0 \\
\hline Perímetro torácico (cm) & $77,8^{a}$ & $75,3^{a}$ & $67,0^{b}$ & $86,0^{a}$ & $76,8^{b}$ & $67,1^{\mathrm{c}}$ \\
\hline Altura al pecho $(\mathrm{cm})$ & $34,0^{\mathrm{b}}$ & $39,7^{a}$ & $33,6^{b}$ & $31,0^{b}$ & $37,4^{a}$ & $33,1^{b}$ \\
\hline Profundidad del pecho (cm) & 28,0 & 28,3 & 25,8 & $35,0^{a}$ & $30,4^{\mathrm{b}}$ & $26,0^{c}$ \\
\hline Longitud cruz-grupa $(\mathrm{cm})$ & $40,0^{a}$ & $37,5^{\mathrm{a}, \mathrm{b}}$ & $36,0^{b}$ & $42,0^{a}$ & $40,8^{a, b}$ & $38,3^{b}$ \\
\hline Largo del lomo (cm) & 18,5 & 17,4 & 16,0 & 17,0 & 16,4 & 15,6 \\
\hline Ancho del lomo (cm) & 13,0 & 12,8 & 12,2 & 13,0 & 12,8 & 12,3 \\
\hline Largo de la pierna (cm) & $30,5^{\mathrm{ab}}$ & $32,2^{a}$ & $28,3^{b}$ & $30,0^{a}$ & $31,2^{\mathrm{a}}$ & $26,6^{b}$ \\
\hline Ancho de la pierna (cm) & 22,6 & 21,3 & 21,0 & $24,0^{a}$ & $22,0^{\mathrm{ab}}$ & $20,1^{b}$ \\
\hline Perímetro de la caña $(\mathrm{cm})$ & 7,3 & 7,4 & 7,0 & 9,0 & 8,0 & 7,4 \\
\hline$\%$ en la muestra & 25,0 & 43,2 & 31,8 & 7,1 & 50,0 & 42,9 \\
\hline
\end{tabular}

abcLiterales distintas en hileras, dentro de sexo, indican diferencias estadísticas $(p<0,05)$.

Las cifras 1,2 y 3 corresponden a los grupos de animales con los valores mayores, intermedios y menores, respectivamente, en las medidas corporales. 


\section{DZIB, ORTIZ DE MONTELLANO Y TORRES-HERNÁNDEZ}

autores han predicho el peso vivo en función del perímetro torácico (Pavon et al., 1986; Fasae et al., 2005; Vilaboa y Díaz Rivera, 2007), longitud de la cabeza y ancho del tronco posterior (Sowande y Sobola, 2008), dimensiones de la grupa (Riva et al., 2004), o bien, con base en un índice a base de combinaciones de medidas, como por ejemplo índice de longitud, índice de profundidad e índice acumulado (Salako, 2006).

Los valores que se muestran en las tablas VI y VII corresponden a un análisis que se hizo con el objetivo de observar qué tanta variabilidad existe en medidas corporales entre animales de uno y tres años de edad. Los animales con los valores mayores se ubican en la categoría uno, aquellos con los valores intermedios en la categoría dos, mientras que los que tienen los menores valores en la tres. Como se puede observar, pocos individuos de un año de edad se ubicaron en la categoría uno $(7,2 \%$ en los machos y $20,5 \%$ en las hembras), mientras que en los animales de tres años de edad esta proporción se revirtió $(30,0 \%$ en machos y $12,0 \%$ en hembras). Esta clasificación permitió detectar una gran variabilidad en las medidas corporales que puede ser utilizada como criterio para una selección temprana, mejoramiento genético y conservación de las características raciales del ovino Blackbelly, ya que la estructura corporal en los animales de un año de edad fue muy cercana a los valores observados en los animales de tres años. A semejanza de ovinos de la raza Segureña de España (Delgado et al., 2004), características como crecimiento, prolificidad y conformación cárnica pueden ser utilizadas como criterios deseables para seleccionar ovinos Blackbelly en México.

En condiciones óptimas de crianza se

Tabla VII. Medias de medidas zoométricas de hembras y machos Blackbelly de tres años de edad. (Means of zoometric measures of Blackbelly ewes and rams three years old).

\begin{tabular}{|c|c|c|c|c|c|c|}
\hline & $1(n=11)^{*}$ & $\begin{array}{l}\text { Hembras } \\
2(n=72)\end{array}$ & $3(n=7)$ & $1(n=3)$ & $\begin{array}{l}\text { Machos } \\
2(n=6)\end{array}$ & $3(n=1)$ \\
\hline Peso vivo $(\mathrm{kg})$ & $46,1^{a}$ & $34,7^{\mathrm{b}}$ & $28,7^{c}$ & $58,5^{a}$ & $46,2^{\mathrm{b}}$ & $45,0^{\mathrm{b}}$ \\
\hline Perímetro testicular (cm) & - & - & - & $30,1^{\mathrm{a}}$ & $30,5^{a}$ & $28,5^{a}$ \\
\hline Altura a la cruz (cm) & $73,0^{\mathrm{a}}$ & $65,7^{b}$ & $61,3^{c}$ & $75,2^{\mathrm{a}}$ & $70,5^{b}$ & $63,0^{c}$ \\
\hline Altura a la grupa (cm) & $71,5^{a}$ & $66,5^{\mathrm{b}}$ & $61,5^{c}$ & $70,0^{a}$ & $68,8^{a}$ & $61,0^{c}$ \\
\hline Ancho del hombro (cm) & $18,1^{a}$ & $16,6^{a, b}$ & $14,9^{b}$ & $19,3^{a}$ & $19,2^{\mathrm{a}}$ & $14,5^{b}$ \\
\hline Ancho del esternón (cm) & 9,8 & 8,9 & 8,1 & 10,2 & 10,2 & 9,5 \\
\hline Perímetro torácico (cm) & $85,6^{a}$ & $77,9^{\mathrm{b}}$ & $70,2^{c}$ & $91,5^{\mathrm{a}}$ & $87,2^{b}$ & $72,0^{c}$ \\
\hline Altura al pecho $(\mathrm{cm})$ & $38,8^{a}$ & $36,5^{a, b}$ & $34,9^{b}$ & $39,5^{\mathrm{a}}$ & $37,0^{a, b}$ & $36,5^{b}$ \\
\hline Profundidad del pecho (cm) & $34,1^{a}$ & $29,1^{b}$ & $26,3^{c}$ & $35,7^{a}$ & $33,4^{\mathrm{a}}$ & $26,5^{b}$ \\
\hline Longitud cruz-grupa $(\mathrm{cm})$ & $42,2^{\mathrm{a}}$ & $39,2^{b}$ & $37,6^{b}$ & $42,1^{b}$ & $38,7^{c}$ & $50,5^{a}$ \\
\hline Largo del lomo (cm) & 18,7 & 17,4 & 16,6 & 18,2 & 18,7 & 18,5 \\
\hline Ancho del lomo (cm) & 13,6 & 12,7 & 12,5 & 15,7 & 14,7 & 16,5 \\
\hline Largo de la pierna (cm) & $32,5^{a}$ & $31,1^{\mathrm{a}, \mathrm{b}}$ & $29,2^{b}$ & $34,8^{a}$ & $32,7^{a}$ & $29,5^{b}$ \\
\hline Ancho de la pierna (cm) & 23,8 & 21,7 & 22,0 & $25,1^{b}$ & $22,5^{c}$ & $28,5^{a}$ \\
\hline Perímetro de la caña $(\mathrm{cm})$ & 8,0 & 7,3 & 7,3 & 9,1 & 8,3 & 9,0 \\
\hline$\%$ en la muestra & 12,2 & 80,0 & 7,8 & 30,0 & 60,0 & 10,0 \\
\hline
\end{tabular}

abc Literales distintas en hileras, dentro de sexo, indican diferencias estadísticas $(p<0,05)$.

Las cifras 1, 2 y 3 corresponden a los grupos de animales con los valores mayores, intermedios y menores, respectivamente, en las medidas corporales.

Archivos de zootecnia vol. 60, núm. 232, p. 1298. 


\section{MEDIDAS CORPORALES DE OVINOS BLACKBELLY EN MÉXICO}

puede estimar el desarrollo de todas las medidas zoométricas y el peso maduro a partir de la medición de un solo componente corporal (Webster, 1989). Sin embargo, en situaciones críticas la variabilidad en el desarrollo de los músculos y la grasa propician que el desarrollo del esqueleto sea la medida más confiable para evaluar cuál es la curva de crecimiento que mejor puede asociarse con un animal en particular (Bocco et al., 2002). Sobre esta base, aunque pueden efectuarse diferentes mediciones del desarrollo del esqueleto, en situaciones productivas comerciales se han popularizado las mediciones del perímetro torácico y altura a la cruz, por su relevancia económica y sencillez operativa (Janssens y Vandepite, 2004).

\section{CONCLUSIÓN}

Existe una alta variabilidad en las medidas corporales de ovinos Blackbelly de

\section{BIBLIOGRAFÍA}

Afolayan, R.A., Adeyinka, I.A. and Lakpini, C.A.M. 2006. The estimation of live weight from body measurements in Yankasa sheep. Czech. J. Anim. Sci., 51: 343-348.

Alderson, G.L.H. 1999. The development of a system of linear measurements to provide an assessment of type and function of beef cattle. AGRI, 25: 45-55.

Álvarez, S., Fresno, M., Capote, J., Delgado, J.V. y Barba, C. 2000a. Estudio para la caracterización de la raza ovina Canaria. Arch. Zootec., 49: 209-215.

Álvarez, S., Fresno, M., Capote, J., Delgado, J.V. y Barba, C. 2000b. Estudio para la caracterización de la raza ovina Palmera. Arch. Zootec., 49: 217-222.

Álvarez Cáceres, R. 2007. Estadística aplicada a las ciencias de la salud. Ediciones Díaz de Santos. España. 778 pp.

Arthur, P.F. and Ahunu, B.K. 1989. Changes in size and shape with time of crossbred West African hair sheep raised under tree-crop plantations. Livest. Prod. Sci., 21: 235-249.

Bocco, O., Bavera, G., Berguer, H. y Petryna, A.
Campeche, México, misma que puede utilizarse para fines de selección. Las diferencias entre machos y hembras en la mayoría de las medidas corporales se expresaron a partir de los dos años de edad. Exceptuando la altura a la grupa en machos y el ancho del esternón y perímetro de la caña en hembras, las medidas corporales aumentaron con la edad ( $\mathrm{p} \leq 0,05)$, alcanzando las hembras su peso maduro y medidas zoométricas más temprano que los machos. De acuerdo con las correlaciones fenotípicas entre las variables analizadas se observa que, al aumentar de peso vivo, los animales de los dos sexos son también más altos y anchos, lo que podría indicar que su tendencia como animales adultos es hacia un animal productor de carne. Por otra parte, las altas y significativas $(p \leq 0,01)$ correlaciones pueden permitir un programa de mejoramiento genético con base en respuestas correlacionadas.

2002. Crecimiento, desarrollo y precocidad. En: Producción bovina de carne. www.produccion bovina.com (03/11/09).

Castillo, H., Román Ponce, H. y Berruecos, J.M. 1974. Características de crecimiento del borrego Tabasco. I. Efecto de la edad y peso al destete y su influencia sobre la fertilidad de la madre. Téc. Pec. Méx., 27: 28-32.

Costa Júnior, G. da S., Guimarães Campelo, J.E., Ribeiro Azevêdo, D.M.M., Filho, R.M., Cavalcante, R.R., Lopes, J.B. and Oliveira, M.E. de. 2006. Morphometric characterization of Santa Inês sheep raised in the regions of Teresina and Campo Maior, Piauí. Rev. Bras. Zootecn., 35: 2260-2267.

Daniel, W.W. 1990. Bioestadística: Base para el análisis de las ciencias de la salud. Editorial Limusa-Noriega. México. 667 pp.

Delgado, J.J., Rodríguez, J.V., León, J.M., Puntas, J., Benavente, M., García, G. y Barba, C. 2004. Esquema de selección de la raza ovina Segureña. Arch. Latinoam. Prod. Anim., 12: 59-62.

Devendra, C. and McLeroy, G.B. 1982. Goat and sheep production in the tropics. Longman Group 


\section{DZIB, ORTIZ DE MONTELLANO Y TORRES-HERNÁNDEZ}

Ltd. New York. pp. 218-219.

Dzib-Can, A., Torres-Hernández, G., Ortíz-deMontellano, A. y Aceves-Navarro, E. 2006. Prácticas de manejo utilizadas por productores de ovinos de pelo de dos sectores sociales en Campeche, México. Livest. Res. Rural Develop., Volumen 18, Artículo \# 105. http://www.Irrd.org/ Irrd18/7/dzib18105.htm (31/08/09).

FA0. 2000. Cada semana se pierden dos razas de animales domésticos. Un nuevo informe lanza la alarma: 1350 razas en peligro de extinción. Comunicado de prensa 00/66. www.fao.org/ WAICENT/OIS/PRESS (12/09/09).

Fasae, O.A., Chineke, A.C. and Alokan, J.A. 2005. Relationship between some physical parameters of grazing Yankasa ewes in the humid zone of Nigeria. Arch. Zootec., 54: 639-642.

González, G.A., Torres-Hernández, G. y Castillo, A.M. 2002. Crecimiento de corderos Blackbelly entre el nacimiento y el peso final en el trópico húmedo de México. Vet. Méx., 33: 443-453.

Gusmão Filho, J.D., Teodoro, S.M., Chaves, M.A. e Oliveira, S.S. 2009. Análise fatorial de medidas morfométricas em ovinos tipo Santa Inês. Arch. Zootec., 58: 289-292.

INEGI. 1998. Instituto Nacional de Geografía e Informática. Anuario Estadístico de Campeche. Campeche. México.

Janssens, S. and Vandepite, W. 2004. Genetic parameters for body measurements and linear type traits in Belgian Bleu du Maine, Suffolk and Texel sheep. Small Ruminant Res., 54: 13-24.

Jonson, D.E. 2000. Métodos multivariados aplicados al análisis de datos. International Thomson Editores. México. pp. 323-384.

Kunene, N., Nesamvuni, E.A. and Fossey, A. 2007. Characterisation of Zulu (Nguni) sheep using linear body measurements and some environmental factors affecting these measurements. S. Afr. J. Anim. Sci., 37: 11-20.

Lastra, M.I. 1998. La importancia de un programa nacional de recursos genéticos pecuarios. Memoria del Tercer Foro de Análisis de los Recursos Genéticos: Ganadería, ovina, caprina, porcina, avícola, apícola, equina y de lidia. SAGAR. México. pp. 1-4.

Levene, H. 1960. Robust tests for the equality of variances. In: I. Olkin, S.G. Ghurye, W. Hoeffding, W.G. Madow, and H.B. Mann (Eds.). Contributions to probability and statistics: Essays in honor of Harold Hotelling. Stanford University Press. pp. 278-292.

Martínez Ávalos, A.M.M., Bores Quintero, R.F. y Castellanos Ruelas, A.F. 1987. Zoometría y predicción de la composición corporal de la borrega Pelibuey. Téc. Pec. Méx., 25: 72-84.

Mason, I.L. 1988. World dictionary of livestock breeds. $3^{\text {rd }}$ Ed. CAB International. New York, NY. USA.

Mehta, B.S., Kandasamy, N. and Arorg, C.L. 1979. Adult body weight of indigenous and crossbred ewes under semi-arid conditions in India. Trop. Anim. Health Pro., 11: 227-230.

Meraz, del Río M., Martínez, V.A.E. y Solís, R.J. 1997. Influencia de los efectos genético-ambientales sobre características de crecimiento de diez genotipos ovinos. Memoria IX Congreso Nacional de Producción Ovina. AMTEO. Querétaro, Qro. México. pp. 20-26.

Mernies, B., Macedo, F., Filonenko, Y. y Fernández, G. 2007. Índices zoométricos en una muestra de ovejas Criollas Uruguayas. Arch. Zootec., 56: 473-478.

Musa, H.H., Suleiman, A.H., El Amin, F.M., Chen, G.H., Mekki, D.M. and Li, B.C. 2005. Evaluation of West African sheep under two production systems. J. Anim. Vet. Adv., 4: 971-975.

Nsoso, S.J., Podisi, B., Otsogile, E., Mokhutshwane, B.S. and Ahmadu, B. 2004. Phenotypic characterization of indigenous Tswana goats and sheep breeds in Botswana: continuous traits. Trop. Anim. Health Pro., 36: 789-800.

Ortiz, O.J., Pacheco, Ch. y Ojeda, C.M. 2000. Crecimiento corporal de machos y hembras Pelibuey a través de medidas zoométricas. Ciclo de Conferencias sobre Evaluación, Comercialización y Mejoramiento Genético. Consejo Nacional de los Recursos Genéticos Pecuarios A.C. Tuxtla, Gutiérrez, Chis. México. pp. 21-25.

Otoikhian, C.S.O., Otoikhian, A.M., Akporhuarho, O.P. and Isidahomen, C. 2008. Correlation of body weight and some body measurement parameters in Ouda sheep under extensive management system. Afr. J. Gen. Agric., 4: 129-133.

Pavon, M., Lima, T. y Ramírez, A. 1986. Medidas corporales de hembras ovinas. Rev. Cubana Reprod. Anim., 12: 7-19.

Peña Blanco, F., García, V.D., Ruíz, F.A. y Mira, M.C. 1990. Estudio biométrico en la raza ovina 


\section{MEDIDAS CORPORALES DE OVINOS BLACKBELLY EN MÉXICO}

Segureña. Arch. Zootec., 39: 249-261.

Riva, J., Rizzi, R., Marelli, S. and Cavalchini, L.G. 2004. Body measurements in Bergamasca sheep. Small Ruminant Res., 55: 221-227.

Ruz, J.G. 1966. Estudio del ovino tropical «Peligüey» del sureste de México y sus cruzas con ovino Merino. Tesis de Licenciatura. Escuela Nacional de Medicina Veterinaria y Zootecnia. Universidad Nacional Autónoma de México. México, D.F. $56 \mathrm{pp}$.

Salako, A.E. 2006. Application of morphological indices in the assessment of type and function in sheep. Int. J. Morphol., 24: 13-18.

Searle, T.W., Graham, N. McC., Donelly, J.B. and Margan, D.E. 1989. Breed and sex differences in skeletal dimensions of sheep in the first year of life. J. Agric. Sci., 113: 349-354.

Sowande, O.S. and Sobola, O.S. 2008. Body measurements of west African dwarf sheep as parameters for estimation of live weight. Trop. Anim. Health Pro., 40: 433-439.

Thys, E. and Hardouin, J. 1991. Prediction of sheep body weight in markets in the far north Cameroon. Livest. Res. Rural Develop., Volumen 3, Artículo \# 1. http://www.Irrd.org//rrd3/1/thys31.htm (23/12/09).

Traoré, A., Tamboura, H.H., Kaboré, A., Royo, L.J., Fernández, I., Álvarez, I., Sangaré, M., Bouchel, D., Poivey, J.P., Francois, D., Toguyeni, A., Sawadogo, L. and Goyache, F. 2008. Multivariate characterization of morphological traits in Burkina Faso sheep. Small Ruminant Res., 80: 62-67.

Vilaboa, A.J. y Díaz Rivera, P. 2007. Conformación corporal de ovinos de pelo. La Revista del Borrego. México, D.F. 8: 38-47.

Webster, A.J.F. 1989. Bioenergetics, bioengineering and growth. Anim. Prod., 48: 249-269. 